\title{
The Interaction Between Self-Reported Sleep Duration and Physical Activity on Peripheral Artery Disease in Chinese Adults: A Cross-Sectional Analysis in the Tianning Cohort Study
}

\author{
Shujing Huang ${ }^{1, *}$ \\ Hongyan Sun ${ }^{2, *}$ \\ Jia $\mathrm{Yu}\left(\mathrm{DI}^{3}\right.$ \\ Hongfei Shi ${ }^{2}$ \\ Liyun Ren ${ }^{3}$ \\ Yan $\mathrm{He}^{3}$ \\ Mingzhi Zhang ${ }^{3}$ \\ Hao Peng $\mathbb{D}^{3,4}$ \\ Heng Guo'
}

'Department of Public Health, School of Medicine, Shihezi University, Shihezi, People's Republic of China; ${ }^{2}$ Center for Disease Prevention and Control of Tianning District, Changzhou, People's Republic of China; ${ }^{3}$ Department of Epidemiology, School of Public Health, Medical College of Soochow University, Suzhou, People's Republic of China; ${ }^{4}$ Jiangsu Key Laboratory of Preventive and Translational Medicine for Geriatric Diseases, Suzhou, People's Republic of China

*These authors contributed equally to this work

Correspondence: Heng Guo

Department of Public Health, School of Medicine, Shihezi University, North 2nd Road, Shihezi, 832000, People's Republic of China

Tel +86993205 7/53

Email shzuguoheng@foxmail.com

Hao Peng

Department of Epidemiology, School of Public Health, Medical College of Soochow University, 199 Renai Road, Industrial Park, Suzhou, 215I23, People's Republic of China

Tel +8651265880078

Fax +8651265880052

Email penghao@suda.edu.cn
Purpose: Sleep duration was associated with large artery atherosclerosis, but its association with atherosclerosis in lower extremity arteries was not well studied. Together with sleep, physical activity constitutes main component of our daily life and influences sleep. Here, we aimed to examine the independent and joint associations of sleep duration and physical activity with peripheral artery disease (PAD) in Chinese adults.

Patients and Methods: In Tianning cohort, night-time sleep duration and physical activity were assessed by the Pittsburgh Sleep Quality Index and the Global Physical Activity Questionnaire, respectively, for 5130 participants $(51.0 \pm 15.6$ years, 58.7\% female). PAD was defined as ankle-brachial index $(\mathrm{ABI})<0.9$. General linear, and logistic regression models were used to assess the associations of sleep duration and physical activity with PAD. The biological interaction between sleep duration and physical activity on PAD was examined using additive model.

Results: Compared to participants sleeping $6-8.9 \mathrm{~h}$, those sleeping $\geq 9 \mathrm{~h}$ had a 0.02 lower ABI $(\beta=-0.02, P=0.007)$ and $38 \%$ higher odds of PAD (OR=1.38, $P=0.035)$. Compared to physically active participants sleeping $6-8.9 \mathrm{~h}$, among $\geq 9 \mathrm{~h}$ group, physically inactive individuals had significantly increased odds of PAD (OR=2.40, $P<0.001)$, whereas physically active individuals did not $(\mathrm{OR}=1.15, P=0.472)$. On additive scale, attributable proportion due to interaction $(0.40,95 \% \mathrm{CI}: 0.07,0.73)$ indicated a significant interaction between sleep duration and physical activity on PAD.

Conclusion: Being physically active may attenuate the detrimental association between prolonged sleep duration and PAD. Moreover, we found a significant interaction between prolonged sleep duration and physical inactivity in the prevalence of PAD.

Keywords: lower extremity disease, Tianning cohort, sleep duration, joint association, physical activity

\section{Introduction}

Peripheral artery disease (PAD), characterized by atherosclerotic obstruction of the arteries in the lower extremities, ${ }^{1}$ is not only a major cardiovascular disease (CVD) but also significantly linked to higher morbidity and mortality of other cardiovascular diseases, representing a considerable public health concern. ${ }^{2,3}$ Decades of efforts have been made regarding its most significant risk factors including obesity, dyslipidemia, hypertension, diabetes, smoking, and chronic kidney disease (CKD), ${ }^{4}$ 
but the prevalence and incidence of PAD continues to be increasing all over the world including China. ${ }^{5,6}$ This phenomenon reminds us that some unknown contributors of PAD may be ignored. Sleep is an essential physiological process that protects our physical and mental health, ${ }^{7}$ and its disruption increases the risks of series of adverse health outcomes, ${ }^{8-11}$ including CVD, through affecting a wide range of physiological processes, eg, glucose metabolism, oxidative stress, and endothelial function, ${ }^{12-15}$ all of which are also involved in the progression of atherosclerosis and PAD. ${ }^{16,17}$ Indeed, short and long sleep duration was found to be associated with coronary artery calcium ${ }^{18-20}$ and carotid intima-media thickness, ${ }^{21,22}$ which are indicators of atherosclerosis in large arteries feeding heart and brain. Nonetheless, as a typical atherosclerotic marker mainly in lower extremity arteries, ankle-brachial index (ABI), which is commonly used to diagnose $\mathrm{PAD},{ }^{23}$ has been poorly studied in association with sleep duration. To date, the association between sleep duration and PAD was only examined by the Multi-Ethnic Study of Atherosclerosis (MESA) which mainly included Caucasian, African, Hispanic, and a small part of Chinese populations. ${ }^{24}$ It's uncertain whether this result could be generalized to Chinese, who have different genetic background and cardiovascular risk profiles. ${ }^{25}$ Therefore, one of the objectives in our study was to examine the association between sleep duration and PAD in an unselected population of Chinese adults.

In addition to sleep, physical activity including occupation, transportation, recreation, and sedentary behavior constitutes another main component of our daily life and is linked to sleep duration. Previous studies reported long sleep duration was related to a low physical activity. ${ }^{26,27}$ Several clinical trials revealed that short sleep duration increased physical activities. ${ }^{28,29}$ Many studies have verified physical activity remained an inverse association with PAD ${ }^{30-32}$ nonetheless there was limited research about this subject in Chinese population. What's more, no study, to our knowledge, has examined the interaction of sleep duration and physical activity on the likelihood of PAD. As such, we aimed to examine the independent and joint associations of sleep duration and physical activity with PAD. We hypothesized improper sleep duration and physical inactivity were associated with PAD respectively, and there was an interaction of improper sleep duration and physical inactivity in PAD.

\section{Materials and Methods}

\section{Study Participants}

The Tianning Cohort is a community-based prospective cohort study which was designed to search for new risk factors and potential therapeutic targets of CVD in Chinese adults. The study design, survey methods, and participants recruitment was approved by the Ethics Committee of Soochow University (approval No. ECSU201800051) and have been detailed elsewhere. ${ }^{33}$ This study was conducted in accordance with the Declaration of Helsinki. Participants aged over 18 years were recruited from 9 randomly selected communities in a traditional but economically developed district Tianning, in the center of Changzhou, China. The methods of participants' recruitment applied in our study were easy to be followed and might relatively reduce the selection bias. After signing a written informed consent, a total of 5199 participants received a face-to-face interview, physical examination, and collection of blood and urine samples between May and November 2018. Of them, participants with missing data on either ABI $(n=14)$ or sleep duration $(n=8)$ were excluded. Participants with an ABI $>1.40$ were also excluded $(n=47)$ because such condition suggests stiff arteries which prevent applicable blood pressure measurements. A total of 5130 participants were included in the final analysis.

\section{Measurement of $A B I$ and Definition of PAD}

Blood pressures of bilateral arm and ankle were measured simultaneously by a four-cuff automated oscillometric device AS-2000 (Hong Kong Biomedical Holdings LTD., Hong Kong, China). After the participants rested for at least 5 minutes in a supine position, the blood pressure measurements were taken twice by study staff who had been centrally trained and certified. The means of the two measurements for each leg and arm were used to calculate the ABI, and the lower value of the two ABIs was used to assess PAD. According to the recommendations of the American Heart Association, ${ }^{23}$ PAD was defined as an $\mathrm{ABI}<0.9$.

\section{Assessment of Sleep Duration}

Sleep duration was assessed by the following question in Pittsburgh Sleep Quality Index (PSQI), ${ }^{34}$ "During the past month, how many hours of actual sleep did you get at 
night? (This may be different than the number of hours you spent in bed)". PSQI was a widely-used and effective self-report questionnaire for assessing sleep quality. PSQIreported sleep duration was found similar to the objectively measured value in prior studies. ${ }^{35,36}$ Consistent with the preceding studies, ${ }^{10,37,38}$ night-time sleep duration was categorized as three groups, $<6 \mathrm{~h}, 6-8.9 \mathrm{~h}, \geq 9 \mathrm{~h}$. Poor sleep quality was defined by $\geq 5$ according to recommendation. ${ }^{39}$

\section{Assessment of Physical Activity}

Physical activity was determined by the Global Physical Activity Questionnaire (GPAQ), which was established by the World Health Organization (WHO) to monitor physical activity in developing countries. ${ }^{40}$ It collects information about physical activity related to work, transportation, recreational activities, and sedentary behavior. According to the GPAQ analysis guidance by $\mathrm{WHO},{ }^{41}$ participants with a MET-min/week $<600$ were considered as physically inactive.

\section{Collection of Risk Factors of PAD}

The methods of data collection have been detailed previously. ${ }^{33}$ In brief, data on education level (years of education), cigarette smoking (current smoking or not), alcohol consumption (current drinking or not) and histories of chronic diseases (including cardiometabolic diseases and obstructive sleep apnea syndrome) were assessed through face-to-face interview by trained staff. The histories of diseases were collected by asking "Has any physician or other medical staff told you that you had certain disease?". Depressive symptom was assessed by the total score of the Center for Epidemiologic Studies Depression Scale (CESD), a widely-used questionnaire in large epidemiological studies. ${ }^{42}$ Physical examinations including body mass index (BMI), systolic (SBP) and diastolic blood pressure (DBP) were performed by trained staff according to standard protocols. Glucose metabolism indicators including fasting plasma glucose and $\mathrm{HbA1c}$, blood lipids including lowdensity lipoprotein cholesterol (LDL-C), high-density lipoprotein cholesterol (HDL-C), total cholesterol (TC), and triglyceride (TG) were measured using commercial reagents (Siemens Healthcare Diagnostic Inc., Co Antrim, UK) at the Key Laboratory of Geriatric Disease Prevention and Translational Medicine of Jiangsu Province. The laboratory staff was unaware of the health status of study participants. Diabetes mellitus was defined by (1) fasting plasma glucose $\geq 7.0 \mathrm{mmol} / \mathrm{L}$; or 2 ) $\mathrm{HbAlc} \geq 6.5 \%$; or 3 ) a self-reported physician diagnosis. ${ }^{43,44}$ Hypertension was defined as meeting any following requirement, (1) SBP $\geq$ $140 \mathrm{mmHg}$; or 2) $\mathrm{DBP} \geq 90 \mathrm{mmHg}$; or 3) a self-reported physician diagnosis. $^{45}$

\section{Statistical Analysis}

Baseline characteristics of participants were presented according to sleep duration levels ( $<6 \mathrm{~h}, 6-8.9 \mathrm{~h}, \geq 9 \mathrm{~h}$ ). Statistical analysis was performed using SPSS 26.0 software (SPSS Inc., Chicago, IL, USA). A two-tailed $P$ value $<0.05$ was considered statistically significant.

\section{Analysis on the Associations of Sleep Duration and Physical Activity with ABI or PAD}

To examine the independent association of sleep duration and physical activity with ABI, we constructed a linear regression model where $\mathrm{ABI}$ was the dependent variable and night-time sleep duration levels/physical activity were/ was the independent variable, adjusting for age, sex, education level, depressive symptom, obstructive sleep apnea syndrome, cigarette smoking, alcohol consumption, BMI, SBP, DBP, diabetes mellitus, TC, LDL-C. To facilitate data interpretation, we similarly constructed a Logistic regression model with the presence of PAD $(y / n)$ as the dependent variable to examine whether sleep duration and physical activity were associated with PAD separately. What's more, we divided the data into two parts according to sex, and repeated the above analysis.

\section{Interaction Analysis}

The joint association of sleep duration and physical activity on PAD was examined based on 6 combinations of sleep duration (categorical as $<6 \mathrm{~h}, 6-8.9 \mathrm{~h}, \geq 9 \mathrm{~h}$ ) and physical activity (categorical as active vs inactive) in a Logistic model, where physically active participants with sleep duration of 6-8.9 $\mathrm{h}$ served as a reference. Furthermore, participants were classified into four groups: participants with either prolonged sleep duration $(\geq 9 \mathrm{~h})$ or physical inactivity (MET-min/week <600), participants with and without both. For the interaction analysis, we constructed a Logistic model setting the above 4 groups as independent variable and PAD as dependent variable. Individuals without prolonged sleep duration and physical inactivity had the lowest prevalence of PAD, therefore were designated as reference group. We applied 3 indexes including the relative excess risk due to interaction 
(RERI), attributable proportion due to interaction (AP), and synergy index (S) to test the significance level of interaction. ${ }^{46,47}$ If there was no interaction, the $95 \%$ confidence intervals (CI) of RERI and AP included 0 , and the $95 \%$ CI of S included 1. The three indexes and their $95 \%$ CIs were calculated by R package "epiR".

\section{Sensitivity Analysis}

To examine whether prevalent CVD and CKD influence the results, participants with these disease histories were excluded.

\section{Results}

\section{Baseline Characteristics of Study}

\section{Participants}

The current study included 5130 participants (mean age: $51.0 \pm 15.6$ years, $58.7 \%$ women). Among them, 531 participants (10.4\%) were suffering from PAD. Their baseline characteristics were shown in Table 1. Participants with prolonged sleep duration ( $\geq 9 \mathrm{~h}$ ) were more likely to be physically inactive, have obstructive sleep apnea syndrome and lower levels of HDL-C and ABI than 6-8.9 h (all $P<0.05$ ). Participants with sleep duration of $<6$ $\mathrm{h}$ tended to be older and have higher level of blood lipids, depressive symptoms, and $\mathrm{ABI}$ than $6-8.9 \mathrm{~h}$ (all $P<0.05$ ).

\section{Associations Between Sleep Duration and Physical Activity with $A B I$ or PAD}

As demonstrated in Tables 2 and 3, compared to participants sleeping 6-8.9 h, those sleeping $\geq 9 \mathrm{~h}$ had a 0.02 lower ABI $(\beta=-0.02, P=0.007)$ and $38 \%$ higher odds of PAD ( $\mathrm{OR}=1.38, P=0.035$ ), adjusting for age, sex, education level, depressive symptom, obstructive sleep apnea syndrome, physical activity, cigarette smoking, alcohol consumption, BMI, SBP, DBP, diabetes mellitus, TC, LDL-C. For men, sleep duration was not associated with $\mathrm{ABI}$ or PAD, on the contrary, women sleeping $\geq 9 \mathrm{~h}$ had a 0.02 lower $\mathrm{ABI}(\beta=-0.02, P=0.041)$ and $51 \%$ higher odds of PAD $(\mathrm{OR}=1.51, P=0.041)$ compared to participants sleeping 6-8.9 $\mathrm{h}$.

As shown in Tables 2 and 3, compared to physically active participants, physically inactive participants had a 0.02 lower $\mathrm{ABI}(\beta=-0.02, P<0.001)$ and $36 \%$ higher odds of PAD (OR=1.36, $P=0.003$ ), adjusting for covariates.

Table I Baseline Characteristics of Participants in Tianning Cohort According to Night-Time Sleep Duration (N=5I30)

\begin{tabular}{|c|c|c|c|c|}
\hline \multirow[t]{2}{*}{ Characteristics } & \multicolumn{3}{|c|}{ Sleep Duration (h/Night) } & \multirow[t]{2}{*}{$P$ value } \\
\hline & $<6$ & $6-8.9$ & $\geq 9$ & \\
\hline No. of participants & 358 & 4319 & 453 & - \\
\hline Sex, men (\%) & $|4|(39.4)$ & $\mid 772(4 \mid .0)$ & $207(45.7)$ & 0.118 \\
\hline Age, years & $55.7 \pm 14.3^{\dagger}$ & $50.7 \pm 15.4$ & $50.2 \pm 18.5^{*}$ & $<0.001$ \\
\hline Education level, years, median (IQR) & $9(8-12)^{\dagger}$ & II (9-14) & $9(9-12)$ & $<0.001$ \\
\hline Cigarette smoking, n (\%) & $64(17.9)$ & $857(19.8)$ & $98(21.6)$ & $0.4 I I$ \\
\hline Alcohol consumption, n (\%) & $68(19.0)$ & $798(18.5)$ & $86(19.0)$ & 0.943 \\
\hline Body mass index, $\mathrm{kg} / \mathrm{m}^{2}$ & $24.01 \pm 3.91$ & $24.08 \pm 3.60$ & $23.78 \pm 3.80$ & 0.232 \\
\hline Systolic blood pressure, $\mathrm{mmHg}$ & $125.6 \pm 16.5$ & $124.0 \pm 16.7$ & $124.8 \pm 18.6$ & 0.180 \\
\hline Diastolic blood pressure, $\mathrm{mmHg}$ & $76.7 \pm 10.4$ & $76.7 \pm 11.2$ & $77.1 \pm 10.4$ & 0.798 \\
\hline Hypertension, n (\%) & $161(45.0)$ & $1686(39.2)$ & $190(42.0)$ & 0.060 \\
\hline Obstructive sleep apnea syndrome, $n$ (\%) & $2(0.6)$ & $29(0.7)$ & $8(1.8)^{\dagger}$ & 0.048 \\
\hline Diabetes mellitus, $\mathrm{n}(\%)$ & $61(17.0)$ & $662(15.4)$ & $59(13.1)$ & 0.291 \\
\hline Total cholesterol, $\mathrm{mmol} / \mathrm{L}$ & $4.94 \pm 0.93^{\dagger}$ & $4.80 \pm 0.94$ & $4.72 \pm 0.89 *$ & 0.003 \\
\hline Triglyceride, $\mathrm{mmol} / \mathrm{L}$ & $1.79 \pm 1.22$ & $1.77 \pm 1.26$ & $1.76 \pm 1.10$ & 0.922 \\
\hline LDL-C, mmol/L & $2.82 \pm 0.80$ & $2.75 \pm 0.79$ & $2.74 \pm 0.78$ & 0.256 \\
\hline HDL-C, mmol/L & $1.23 \pm 0.32^{\dagger}$ & $1.22 \pm 0.31$ & $1.18 \pm 0.28 * \dagger$ & 0.013 \\
\hline Depressive symptom, points, median (IQR) & $\mathrm{I}(0-12)^{\dagger}$ & $0(0-5)$ & $0(0-5)^{*}$ & $<0.001$ \\
\hline Physically active, $n$ (\%) & $285(79.6)$ & $3236(74.9)$ & $310(68.4)^{* \dagger}$ & 0.001 \\
\hline Ankle branchial index & $1.09 \pm 0.13^{\dagger}$ & $1.08 \pm 0.15$ & $1.06 \pm 0.17^{* \dagger}$ & 0.003 \\
\hline Peripheral artery disease, $\mathrm{n}(\%)$ & $31(8.7)$ & $438(10.1)$ & 62 (13.7) & 0.034 \\
\hline
\end{tabular}

Notes: ${ }^{\dagger} P<0.05$ compared with participants with a sleep duration of $6-8.9$ hours. $* P<0.05$ compared with participants with a sleep duration of $<6$ hours. Results presented as mean \pm standard deviation, unless otherwise noted. Bolding represented $P<0.05$.

Abbreviations: LDL-C, low density lipoprotein cholesterol; HDL-C, high density lipoprotein cholesterol; IQR, interquartile range. 
Table 2 The Multivariate Adjusted Associations of Sleep Duration and Physical Activity with ABI

\begin{tabular}{|c|c|c|c|c|c|c|c|}
\hline \multirow[t]{2}{*}{ Variables } & \multicolumn{3}{|c|}{ Overall } & \multicolumn{2}{|c|}{ Men } & \multicolumn{2}{|c|}{ Women } \\
\hline & Mean \pm SD & $\beta(\mathrm{SE})^{\mathrm{a}}$ & $P$ value & $\beta(\mathrm{SE})^{\mathrm{a}}$ & $P$ value & $\beta(\mathrm{SE})^{\mathrm{a}}$ & $P$ value \\
\hline $\begin{array}{l}\text { Sleep duration }(\mathrm{h} / \mathrm{night}) \\
<6 \\
6-8.9 \\
\geq 9\end{array}$ & $\begin{array}{l}1.09 \pm 0.13 \\
1.08 \pm 0.15 \\
1.06 \pm 0.17\end{array}$ & $\begin{array}{l}0.01(0.01) \\
\operatorname{Ref} \\
-0.02(0.01)\end{array}$ & $\begin{array}{l}0.433 \\
- \\
\mathbf{0 . 0 0 7}\end{array}$ & $\begin{array}{l}0.00(0.01) \\
\operatorname{Ref} \\
-0.02(0.01)\end{array}$ & $\begin{array}{l}0.957 \\
- \\
0.122\end{array}$ & $\begin{array}{l}0.01(0.01) \\
\text { Ref } \\
-0.02(0.01)\end{array}$ & $\begin{array}{l}0.338 \\
- \\
\mathbf{0 . 0 4 1}\end{array}$ \\
\hline $\begin{array}{l}\text { Physical activity } \\
\text { Active } \\
\text { Inactive }\end{array}$ & $\begin{array}{l}1.08 \pm 0.15 \\
1.06 \pm 0.16\end{array}$ & $\begin{array}{l}\text { Ref } \\
-0.02(0.01)\end{array}$ & $\begin{array}{l}- \\
<0.001\end{array}$ & $\begin{array}{l}\text { Ref } \\
-0.03(0.01)\end{array}$ & $\begin{array}{l}- \\
0.001\end{array}$ & $\begin{array}{l}\text { Ref } \\
-0.02(0.01)\end{array}$ & $\begin{array}{l}- \\
0.001\end{array}$ \\
\hline
\end{tabular}

Notes: ${ }^{a}$ Adjusted for age, sex, education level, depressive symptom, obstructive sleep apnea syndrome, cigarette smoking, alcohol consumption, body mass index, systolic blood pressure, diastolic blood pressure, diabetes mellitus, total cholesterol, low-density lipoprotein cholesterol. Bolding represented $P<0.05$.

Abbreviations: $A B I$, ankle-brachial index; $P A D$, peripheral artery disease.

Table 3 The Multivariate Adjusted Associations of Sleep Duration and Physical Activity with PAD

\begin{tabular}{|c|c|c|c|c|c|c|c|}
\hline \multirow[t]{2}{*}{ Variables } & \multicolumn{3}{|c|}{ Overall } & \multicolumn{2}{|c|}{ Men } & \multicolumn{2}{|c|}{ Women } \\
\hline & $\mathbf{N}(\%)$ & OR $(95 \% \mathrm{CI})^{\mathrm{a}}$ & $P$ value & OR $(95 \% \mathrm{CI})^{\mathrm{a}}$ & $P$ value & OR $(95 \% \mathrm{Cl})^{\mathrm{a}}$ & $P$ value \\
\hline $\begin{array}{l}\text { Sleep duration (h/night) } \\
<6 \\
6-8.9 \\
\geq 9\end{array}$ & $\begin{array}{l}31(8.7) \\
438(10.1) \\
62(13.7)\end{array}$ & $\begin{array}{l}0.87(0.58,1.30) \\
\operatorname{Ref} \\
I .38(1.02,1.85)\end{array}$ & $\begin{array}{l}0.535 \\
- \\
\mathbf{0 . 0 3 5}\end{array}$ & $\begin{array}{l}0.75(0.38,1.46) \\
\operatorname{Ref} \\
1.22(0.78,1.92)\end{array}$ & $\begin{array}{l}0.398 \\
- \\
0.385\end{array}$ & $\begin{array}{l}0.98(0.58,1.63) \\
\operatorname{Ref} \\
I .5 \mathrm{I}(1.02,2.24)\end{array}$ & $\begin{array}{l}0.921 \\
- \\
\mathbf{0 . 0 4 1}\end{array}$ \\
\hline $\begin{array}{l}\text { Physical activity } \\
\text { Active } \\
\text { Inactive }\end{array}$ & $\begin{array}{l}365(9.5) \\
166(12.8)\end{array}$ & $\begin{array}{l}\text { Ref } \\
1.36(1.11,1.67)\end{array}$ & $\begin{array}{l}- \\
0.003\end{array}$ & $\begin{array}{l}\text { Ref } \\
1.47(1.08,2.02)\end{array}$ & $\begin{array}{l}- \\
0.016\end{array}$ & $\begin{array}{l}\text { Ref } \\
1.29(0.99,1.68)\end{array}$ & $\begin{array}{l}- \\
0.062\end{array}$ \\
\hline
\end{tabular}

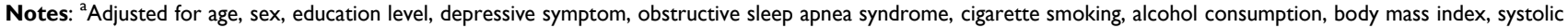
blood pressure, diastolic blood pressure, diabetes mellitus, total cholesterol, low-density lipoprotein cholesterol. Bolding represented $P<0.05$.

Abbreviations: $A B I$, ankle-brachial index; $P A D$, peripheral artery disease.

Either for men $(\beta=-0.03, P=0.001)$ or women $(\beta=-0.02$, $P=0.001)$, physical inactivity was associated with lower ABI. For both genders, physical inactivity was related with higher prevalence of $\mathrm{PAD}$, however, the association in women was not statistically significant.

Additionally, we did the analysis of sleep quality and multi-dimensional sleep disorders offered by Pittsburgh questionnaire with $\mathrm{ABI}$ or $\mathrm{PAD}$, however, most of data was unsignificant. We included this analysis in Supplementary Tables (Table S1).

\section{Interaction of Sleep Duration and Physical Activity on the Prevalence of PAD}

Compared to physically active participants sleeping 6$8.9 \mathrm{~h}$, among $\geq 9 \mathrm{~h}$ group, physically inactive individuals had significantly increased odds of PAD $(\mathrm{OR}=2.40$, $P<0.001)$, whereas physically active individuals did not $(\mathrm{OR}=1.15, P=0.472$ ) (Figure 1). We further examined the interaction between prolonged sleep duration and physical inactivity on PAD. As shown in Table 4, among participants in 4 different combinations, PAD was the most common in participants with both prolonged sleep duration and physical inactivity with a prevalence of $18.9 \%$. Similarly, after adjustment for covariates, the OR $(2.41, P<0.001)$ was much higher for participants with both prolonged sleep duration and physical inactivity than participants with prolonged sleep duration only $(\mathrm{OR}=1.16, P=0.450)$ and participants with physical inactivity only $(\mathrm{OR}=1.29, P=0.022)$. On additive scale, AP $(0.40,95 \%$ CI: $0.07,0.73)$ was found significant, and it indicated there was about $40 \%$ of the higher prevalence of PAD in participants with prolonged sleep duration and physical inactivity attributed to the interaction between sleep duration and physical activity (Table 4). On multiplicative scale, the results did not indicate a significant interaction. 


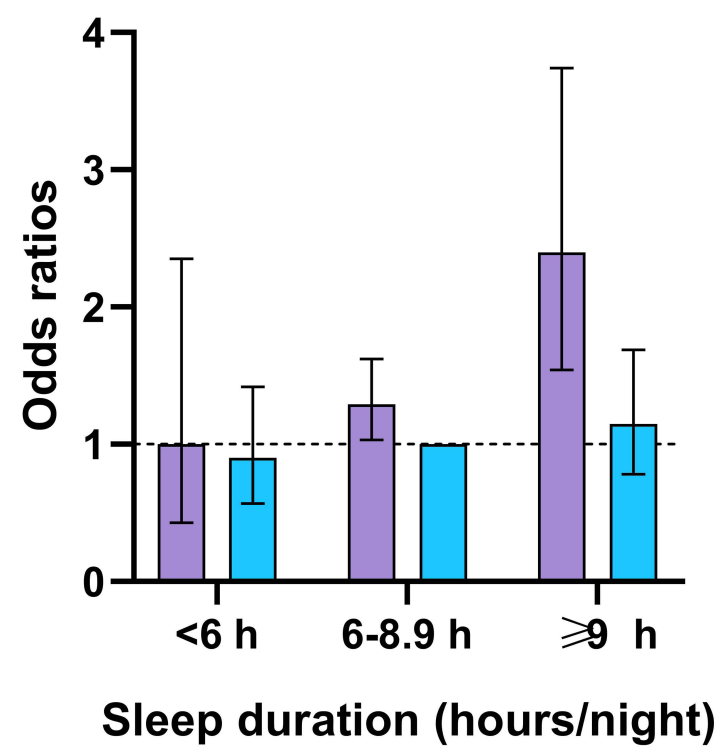

\section{Physically inactive \\ Physically active}

Figure I The joint association of sleep duration and physical activity on PAD. ORs and $95 \%$ Cls of PAD were calculated setting physically active participants with 6-8.9 hours/night of sleep duration as the reference, adjusting age, sex, education level, depressive symptom, obstructive sleep apnea syndrome, cigarette smoking, alcohol consumption, body mass index, systolic blood pressure, diastolic blood pressure, diabetes mellitus, total cholesterol, low-density lipoprotein cholesterol.

\section{Sensitivity Analysis}

After excluding participants with CVD and CKD (N=313), the association of sleep duration and physical activity with PAD did not change (Table S2). Besides, the results of interaction between sleep duration and physical activity on PAD kept in line with those before (Table S3).

\section{Discussion}

In this large-scale community-based study including over 5000 Chinese adults, we found prolonged sleep duration $(\geq 9 \mathrm{~h}$ ) and physical inactivity (MET-min/week $<600$ ) were independently and significantly associated with higher prevalence of PAD. However, prolonged sleep duration was related with ABI or PAD only in women. Further, being physically active may attenuate the detrimental association between prolonged sleep duration and PAD, and there was a significant interaction between prolonged sleep duration and physical inactivity in the prevalence of PAD. Our results suggest that, for individuals with prolonged sleep duration, being physically active might reduce the lesion in lower extremity artery. Our study also highlights the importance of both reasonable sleep duration and being physically active for PAD in the clinical and preventive medicine domains.

In our study, the mean of sleep duration was 7.2 hours per night. Similarly, a community-based study reported the mean sleep duration of 7.9 hours per night in different geographical regions of China. ${ }^{48}$ Together with our study, MESA reported long sleep duration $(>8 \mathrm{~h}$ ) was related with increased odds of PAD. ${ }^{24} \mathrm{~A}$ meta-analysis involving 5,134,036 participants from prospective cohort studies found a significant linear association between longer

Table 4 The Interaction Between Sleep Duration and Physical Activity in the Prevalence of PAD

\begin{tabular}{|c|c|c|c|c|}
\hline \multirow[t]{3}{*}{ Prolonged Sleep Duration } & \multicolumn{4}{|c|}{ Physical Inactivity } \\
\hline & \multicolumn{2}{|c|}{$(-)$} & \multicolumn{2}{|r|}{$(+)$} \\
\hline & N with PAD (\%) & OR (95\% Cl); P & N with PAD (\%) & OR (95\% Cl); P \\
\hline$(-)$ & $330(9.4)$ & Ref & $139(12.0)$ & $1.29(1.04,1.60) ; P=\mathbf{0 . 0 2 2}$ \\
\hline$(+)$ & $35(11.3)$ & $1.16(0.79, I .7 I) ; P=0.450$ & 27 (18.9) & $2.4 \mathrm{I}(\mathrm{I} .55,3.76) ; P<0.00 \mathrm{I}$ \\
\hline
\end{tabular}

Notes: Measure of interaction on additive scale: RERI $(95 \% \mathrm{Cl})=0.96(-0.18,2.1 \mathrm{I})$; AP $(95 \% \mathrm{Cl})=0.40(0.07,0.73)$; S $(95 \% \mathrm{Cl})=3.14(0.79, \mathrm{I} 2.46)$. Measure of interaction on multiplicative scale: OR $(95 \% \mathrm{Cl})=1.50(0.83,2.7 \mathrm{I})$. ORs were adjusted for age, sex, education level, depressive symptom, obstructive sleep apnea syndrome, cigarette smoking, alcohol consumption, body mass index, systolic blood pressure, diastolic blood pressure, diabetes mellitus, total cholesterol, low-density lipoprotein cholesterol. Bolding represented $P<0.05$.

Abbreviation: PAD, peripheral artery disease. 
sleep duration and incident cardiovascular disease. ${ }^{49}$ Our findings support a growing body of evidence that prolonged sleep duration was associated with higher odds of atherosclerosis and CVD. Nonetheless, different from our findings, a study indicated very short sleep duration $(<6 \mathrm{~h})$ was associated with a higher atherosclerotic burden in the carotid and femoral arteries. ${ }^{7}$ Another study found short sleep duration $(<6 \mathrm{~h})$ was associated with the presence of coronary calcium (coronary calcium score $>0$ ). ${ }^{50}$ We did not observe a significant association between short sleep duration and PAD in our study. This phenomenon may due to different study populations and objectives.

Further, sex difference was found in the association of sleep duration with ABI or PAD. Consistently, a study found long sleep duration was related with higher atherosclerotic burden specifically in women. ${ }^{7}$ As for the association between sleep duration and arterial stiffness, the conclusions were ambiguous, one study only for men, ${ }^{38}$ another only for women. ${ }^{51}$ Little was known about the optimal sleep duration for both gender, and more studies were warranted to investigate the underlying mechanism. ${ }^{52}$

In addition to sleep, physical activity makes up another major part in our daily life and is related to sleep duration. Consistent with the findings mentioned in the introduction, our results showed participants sleeping $\geq 9 \mathrm{~h}$ were more likely to be physically inactive than $6-8.9 \mathrm{~h}$ in the baseline analysis. Same as previous studies, ${ }^{30-32}$ our results revealed that being physically active was related with lower $\mathrm{ABI}$ and higher odds of PAD. However, the present study was based on a cross-sectional design, therefore, we were unable to clarify the directions of above associations. To be detailed, we could not confirm whether prolonged sleep duration and physical inactivity were causes or symptoms of PAD.

Moreover, we found physical activity might mitigate the association between prolonged sleep duration and higher odds of PAD. As mentioned earlier, the joint association of sleep duration and physical activity with PAD has not been reported before. However, in alignment with our findings, some evidences have indicated physical activity may attenuate the adverse relations between improper sleep durations and several cardiometabolic factors. For example, a British study revealed higher physical activity may attenuate the detrimental associations of unreasonable sleep duration $(<7 \mathrm{~h}$ or $>9 \mathrm{~h}$ ) with BMI, blood lipids, hypertension, and diabetes. ${ }^{53}$ Another 15year follow-up cohort study including 341,248 Chinese demonstrated being physically active (MET-min/week $\geq 900$ ) could eliminate the risk of CVD mortality in long sleep duration $\left(>8\right.$ h) group. $^{54}$ Moreover, our study supported a significant interaction between prolonged sleep duration and physical inactivity in the prevalence of PAD. Similarly, a cross-sectional study demonstrated a joint association of short sleep duration $(\leq 7 \mathrm{~h})$ and low physical activity with insulin resistance among non-diabetic Chinese adults. ${ }^{55}$ Another longitudinal Chinese study revealed participants with poor sleep (sleeping $<7 \mathrm{~h}$ or $>9 \mathrm{~h}$ ) and low physical activity had the highest multimorbidity of chronic diseases compared to other combinations. ${ }^{56}$

CVD and CKD kept a close relationship with PAD, and individuals with CVD or CKD might experience longer sleep duration than the normal. ${ }^{57,58}$ Therefore, we did a sensitivity analysis to see whether participants with a history of CVD or CKD affect the results. Finally, we found excluding participants with CVD and CKD did not change previous results. The results indicated that the independent and joint associations of sleep duration and physical activity with PAD were not driven by a history of CVD and CKD. The sensitivity analysis revealed our results were robust and credible.

The underlying mechanism linking prolonged sleep duration to PAD was not clear. ${ }^{18,24} \mathrm{He}$ et al ${ }^{59}$ reported long but not short sleep duration was independently associated with elevated high-sensitivity C-reactive protein levels. Physical inactivity ${ }^{30}$ was associated with inflammation responses, ${ }^{60,61}$ endothelial function, ${ }^{62}$ oxidative stress. ${ }^{63}$ These above pathologic processes were involved in the progression of PAD. ${ }^{16,17}$ Additionally, both prolonged sleep duration ${ }^{9}$ and physical inactivity ${ }^{64-66}$ were found to be positively associated with obesity, diabetes, and hypertension, all of which were risk factors of PAD. The above mechanisms explained the associations of prolonged sleep duration and physical inactivity with PAD.

To the best of our knowledge, our study was the first to verify the association of sleep duration or physical activity with PAD in Chinese, and to examine the interaction between sleep duration and physical activity in PAD. The strengths of this study included a relatively large sample size, comprehensive adjustment for conventional risk factors, and careful analysis of the interaction between sleep duration and physical activity in PAD. However, some limitations should be acknowledged in our study. First, only cross-sectional data was included, as such the casual relationship of sleep duration and physical activity with PAD was not elucidated. Second, although we used unselected sample, the population in the Tianning Cohort was the Han nationality and could not represent other ethnic/racial populations. Additionally, only selfreported questionnaires were used to assess sleep duration and 
physical activity, therefore misclassification and recall bias might exist and influence the results. Thus, the generalizability of our results should be taken cautiously.

\section{Conclusion}

In summary, we found being physically active may attenuate the detrimental association between prolonged sleep duration and PAD. Moreover, the results indicated a significant interaction between prolonged sleep duration and physical inactivity in the prevalence of PAD. Prospective studies and randomized clinical trials are warranted to examine the casual relationship of sleep duration and physical activity with PAD and the interaction of sleep duration and physical activity in cardiovascular health.

\section{Abbreviations}

PAD, peripheral artery disease; CVD, cardiovascular disease; CKD, chronic kidney disease; ABI, ankle-brachial index; MESA, the Multi-Ethnic Study of Atherosclerosis; PSQI, Pittsburgh Sleep Quality Index; GPAQ, Global Physical Activity Questionnaire; MET, metabolic equivalent; WHO, World Health Organization; CES-D, the Center for Epidemiologic Studies Depression Scale; BMI, body mass index; SBP, systolic blood pressure; DBP, diastolic blood pressure; LDL-C, low-density lipoprotein cholesterol; HDL-C, high-density lipoprotein cholesterol; TC, total cholesterol; TG triglyceride.

\section{Data Sharing Statement}

The data are available on request from the corresponding author at penghao@suda.edu.cn.

\section{Acknowledgments}

We appreciate the participants in this study and all staffs a lot for their support and assistance. Especially, we thank The Center for Disease Prevention and Control of Tianning District for their support in the recruitment of participants.

\section{Funding}

This work has been funded by the National Natural Science Foundation of China (NO. 81903384 and 81872690), the Natural Science Foundation of Jiangsu Province (NO. BK20180841), the Suzhou Municipal Science and Technology Bureau (NO. SS201853 and SYS2020091), a Project of the Priority Academic Program Development of Jiangsu Higher Education Institutions, and the Science and Technology Project of
Xinjiang Production and Construction Corps (NO. 2021AB030). The funders had no role in study design, data collection and analysis, decision to publish, or preparation of the manuscript.

\section{Disclosure}

The authors report no conflicts of interest in this work.

\section{References}

1. Criqui MH, Aboyans V. Epidemiology of peripheral artery disease. Circ Res. 2015;116(9):1509-1526. doi:10.1161/CIRCRESAHA.1 16.303849

2. Golomb BA, Dang TT, Criqui MH. Peripheral arterial disease: morbidity and mortality implications. Circulation. 2006;114(7):688-699. doi:10.1161/CIRCULATIONAHA.105.593442

3. Song P, Rudan D, Zhu Y, et al. Global, regional, and national prevalence and risk factors for peripheral artery disease in 2015: an updated systematic review and analysis. Lancet Glob Health. 2019;7 (8):e1020-e1030. doi:10.1016/S2214-109X(19)30255-4

4. Fowkes FG, Aboyans V, Fowkes FJ, McDermott MM, Sampson UK, Criqui MH. Peripheral artery disease: epidemiology and global perspectives. Nat Rev Cardiol. 2017;14(3):156-170. doi:10.1038/ nrcardio.2016.179

5. Fowkes FG, Rudan D, Rudan I, et al. Comparison of global estimates of prevalence and risk factors for peripheral artery disease in 2000 and 2010: a systematic review and analysis. Lancet. 2013;382 (9901):1329-1340. doi:10.1016/S0140-6736(13)61249-0

6. Song P, Rudan D, Wang M, Chang X, Rudan I. National and subnational estimation of the prevalence of peripheral artery disease (PAD) in China: a systematic review and meta-analysis. $J$ Glob Health. 2019;9(1):010601. doi:10.7189/jogh.09.010601

7. Domínguez F, Fuster V, Fernández-Alvira JM, et al. Association of sleep duration and quality with subclinical atherosclerosis. $\mathrm{J}$ Am Coll Cardiol. 2019;73(2):134-144. doi:10.1016/j.jacc.2018.10.060

8. Ayas NT, White DP, Al-Delaimy WK, et al. A prospective study of self-reported sleep duration and incident diabetes in women. Diabetes Care. 2003;26(2):380-384. doi:10.2337/diacare.26.2.380

9. Buxton OM, Marcelli E. Short and long sleep are positively associated with obesity, diabetes, hypertension, and cardiovascular disease among adults in the United States. Soc Sci Med. 2010;71 (5):1027-1036. doi:10.1016/j.socscimed.2010.05.041

10. Cappuccio FP, Cooper D, D’Elia L, Strazzullo P, Miller MA. Sleep duration predicts cardiovascular outcomes: a systematic review and meta-analysis of prospective studies. Eur Heart J. 2011;32 (12):1484-1492. doi:10.1093/eurheartj/ehr007

11. Li J, Huang Z, Hou J, et al. Sleep and CKD in Chinese adults: a cross-sectional study. Clin J Am Soc Nephrol. 2017;12(6):885-892. doi:10.2215/CJN.09270816

12. Buxton OM, Pavlova M, Reid EW, Wang W, Simonson DC, Adler GK. Sleep restriction for 1 week reduces insulin sensitivity in healthy men. Diabetes. 2010;59(9):2126-2133. doi:10.2337/db09-0699

13. Carreras A, Zhang SX, Peris E, et al. Chronic sleep fragmentation induces endothelial dysfunction and structural vascular changes in mice. Sleep. 2014;37(11):1817-1824. doi:10.5665/sleep.4178

14. Hill VM, O'Connor RM, Sissoko GB, et al. A bidirectional relationship between sleep and oxidative stress in Drosophila. PLoS Biol. 2018;16(7):e2005206. doi:10.1371/journal.pbio.2005206

15. Spiegel K, Tasali E, Penev P, Van Cauter E. Brief communication: sleep curtailment in healthy young men is associated with decreased leptin levels, elevated ghrelin levels, and increased hunger and appetite. Ann Intern Med. 2004;141(11):846-850. doi:10.7326/ 0003-4819-141-11-200412070-00008 
16. Signorelli SS, Scuto S, Marino E, Xourafa A, Gaudio A. Oxidative stress in Peripheral Arterial Disease (PAD) mechanism and biomarkers. Antioxidants (Basel). 2019;8(9):367.

17. Yang SL, Zhu LY, Han R, Sun LL, Li JX, Dou JT. Pathophysiology of peripheral arterial disease in diabetes mellitus. J Diabetes. 2017;9 (2):133-140. doi:10.1111/1753-0407.12474

18. Kim CW, Chang Y, Zhao D, et al. Sleep duration, sleep quality, and markers of subclinical arterial disease in healthy men and women. Arterioscler Thromb Vasc Biol. 2015;35(10):2238-2245. doi:10.1161/ ATVBAHA.115.306110

19. King CR, Knutson KL, Rathouz PJ, Sidney S, Liu K, Lauderdale DS. Short sleep duration and incident coronary artery calcification. JAMA. 2008;300(24):2859-2866.

20. Matthews KA, Strollo PJ Jr., Hall M, et al. Associations of Framingham risk score profile and coronary artery calcification with sleep characteristics in middle-aged men and women: Pittsburgh SleepSCORE study. Sleep. 2011;34(6):711-716.

21. Ma CC, Burchfiel CM, Charles LE, et al. Associations of objectively measured and self-reported sleep duration with carotid artery intima media thickness among police officers. Am J Ind Med. 2013;56 (11):1341-1351.

22. Sands MR, Lauderdale DS, Liu K, et al. Short sleep duration is associated with carotid intima-media thickness among men in the Coronary Artery Risk Development in Young Adults (CARDIA) study. Stroke. 2012;43(11):2858-2864. doi:10.1161/STROKEAHA.1 12.660332

23. Aboyans V, Criqui MH, Abraham P, et al. Measurement and interpretation of the ankle-brachial index: a scientific statement from the American Heart Association. Circulation. 2012;126(24):2890-2909. doi:10.1161/CIR.0b013e318276fbcb

24. Nagayoshi M, Lutsey PL, Benkeser D, et al. Association of sleep apnea and sleep duration with peripheral artery disease: the Multi-Ethnic Study of Atherosclerosis (Mesa). Atherosclerosis. 2016;251:467-475. doi:10.1016/j.atherosclerosis.2016.06.040

25. Pang Y, Lyu J, Yu C, Guo Y, Lee L. Risk factors for cardiovascular disease in the Chinese population: recent progress and implications. Glob Health J. 2020;4(3):65-71. doi:10.1016/j.glohj.2020.08.004

26. Stranges S, Dorn JM, Shipley MJ, et al. Correlates of short and long sleep duration: a cross-cultural comparison between the United Kingdom and the United States: the Whitehall II Study and the Western New York Health Study. Am J Epidemiol. 2008;168 (12):1353-1364. doi:10.1093/aje/kwn337

27. Savin KL, Patel SR, Clark TL, et al. Relationships of Sleep Duration, Midpoint, and Variability with Physical Activity in the HCHS/SOL Sueño Ancillary Study. Behav Sleep Med. 2020;19:1-12.

28. Tajiri E, Yoshimura E, Hatamoto Y, Tanaka H, Shimoda S. Effect of sleep curtailment on dietary behavior and physical activity: a randomized crossover trial. Physiol Behav. 2018;184:60-67. doi:10.1016/j.physbeh.2017.11.008

29. Brondel L, Romer MA, Nougues PM, Touyarou P, Davenne D. Acute partial sleep deprivation increases food intake in healthy men. $\mathrm{Am}$ J Clin Nutr. 2010;91(6):1550-1559. doi:10.3945/ajcn.2009.28523

30. Stein RA, Rockman CB, Guo Y, et al. Association between physical activity and peripheral artery disease and carotid artery stenosis in a self-referred population of 3 million adults. Arterioscler Thromb Vasc Biol. 2015;35(1):206-212. doi:10.1161/ATVBAHA.114.304161

31. Heikkilä K, Coughlin PA, Pentti J, Kivimäki M, Halonen JI. Physical activity and peripheral artery disease: two prospective cohort studies and a systematic review. Atherosclerosis. 2019;286:114-120. doi:10.1016/j.atherosclerosis.2019.05.008

32. Bertoni AG, Whitt-Glover MC, Chung $\mathrm{H}$, et al. The association between physical activity and subclinical atherosclerosis: the Multi-Ethnic Study of Atherosclerosis. Am J Epidemiol. 2009;169 (4):444-454. doi:10.1093/aje/kwn350
33. Yu J, Sun H, Shang F, et al. Association between glucose metabolism and vascular aging in Chinese adults: a cross-sectional analysis in the Tianning Cohort Study. Clin Interv Aging. 2019;14:1937-1946. doi: $10.2147 /$ CIA.S223690

34. Buysse DJ, Reynolds CF 3rd, Monk TH, Berman SR, Kupfer DJ. The Pittsburgh Sleep Quality Index: a new instrument for psychiatric practice and research. Psychiatry Res. 1989;28(2):193-213. doi:10.1016/0165-1781(89)90047-4

35. Landry GJ, Best JR, Liu-Ambrose T. Measuring sleep quality in older adults: a comparison using subjective and objective methods. Front Aging Neurosci. 2015;7:166. doi:10.3389/fnagi.2015.00166

36. Lauderdale DS, Knutson KL, Yan LL, Liu K, Rathouz PJ. Self-reported and measured sleep duration: how similar are they? Epidemiology. 2008;19(6):838-845. doi:10.1097/EDE.0b013e318187a7b0

37. Heslop P, Smith GD, Metcalfe C, Macleod J, Hart C. Sleep duration and mortality: the effect of short or long sleep duration on cardiovascular and all-cause mortality in working men and women. Sleep Med. 2002;3(4):305-314. doi:10.1016/S1389-9457(02)00016-3

38. Tsai TC, Wu JS, Yang YC, Huang YH, Lu FH, Chang CJ. Long sleep duration associated with a higher risk of increased arterial stiffness in males. Sleep. 2014;37(8):1315-1320. doi:10.5665/sleep.3920

39. Jennings JR, Muldoon MF, Hall M, Buysse DJ, Manuck SB. Selfreported sleep quality is associated with the metabolic syndrome. Sleep. 2007;30(2):219-223. doi:10.1093/sleep/30.2.219

40. Armstrong T, Bull F. Development of the World Health Organization Global Physical Activity Questionnaire (GPAQ). J Public Health (Bangkok). 2006;14(2):66-70. doi:10.1007/s10389-006-0024-x

41. World Health Organization. Global Physical Activity Questionnaire (GPAQ) analysis guide. Available from: https:/www.who.int/ncds/ surveillance/steps/resources/GPAQ_Analysis_Guide.pdf. Accessed September 18, 2021.

42. Radloff LS. The CES-D scale: a self-report depression scale for research in the general population. Appl Psychol Meas. 1977;1 (3):385-401. doi:10.1177/014662167700100306

43. American Diabetes Association. Diagnosis and classification of diabetes mellitus. Diabetes Care. 2011;34(Supp11):S62-S69. doi:10.2337/dc11-S062

44. Yuan X, Liu T, Wu L, Zou ZY, Li C. Validity of self-reported diabetes among middle-aged and older Chinese adults: the China Health and Retirement Longitudinal Study. BMJ Open. 2015;5(4):e006633. doi:10.1136/bmjopen-2014-006633

45. Liu LS, Wu ZS, Wang JG, et al. 2018 Chinese guidelines for prevention and treatment of hypertension-a report of the Revision Committee of Chinese Guidelines for prevention and treatment of hypertension. J Geriatr Cardiol. 2019;16(3):182-241.

46. Knol MJ, VanderWeele TJ. Recommendations for presenting analyses of effect modification and interaction. Int $J$ Epidemiol. 2012;41(2):514-520. doi:10.1093/ije/dyr218

47. Hallqvist J, Ahlbom A, Diderichsen F, Reuterwall C. How to evaluate interaction between causes: a review of practices in cardiovascular epidemiology. J Intern Med. 1996;239(5):377-382. doi:10.1046/ j.1365-2796.1996.431782000.x

48. Zheng R, Niu J, Wu S, et al. Gender and age differences in the association between sleep characteristics and fasting glucose levels in Chinese adults. Diabetes Metab. 2021;47(2):101174. doi:10.1016/ j.diabet.2020.07.001

49. Jike M, Itani O, Watanabe N, Buysse DJ, Kaneita Y. Long sleep duration and health outcomes: a systematic review, meta-analysis and meta-regression. Sleep Med Rev. 2018;39:25-36. doi:10.1016/j. smrv.2017.06.011

50. Blasco-Colmenares E, Moreno-Franco B, Latre ML, et al. Sleep duration and subclinical atherosclerosis: the Aragon Workers' Health Study. Atherosclerosis. 2018;274:35-40. doi:10.1016/j. atherosclerosis.2018.05.003 
51. Liu X, Song Q, Wu S, Wang X. Long sleep duration and risk of increased arterial stiffness in a Chinese population. Medicine (Baltimore). 2020;99(36):e22073. doi:10.1097/MD.00000000 00022073

52. Kwon S, Lee H, Lee JT, Shin MJ, Choi S, Oh H. Sleep duration and mortality in Korean adults: a population-based prospective cohort study. BMC Public Health. 2020;20(1):1623. doi:10.1186/s12889020-09720-3

53. Huang BH, Inan-Eroglu E, Hamer M, Stamatakis E. Joint associations of device-measured physical activity and sleep duration with cardiometabolic health in the 1970 British Cohort Study. J Sci Med Sport. 2020;23(12):1191-1196. doi:10.1016/j.jsams.2020.07.012

54. Chen LJ, Hamer M, Lai YJ, Huang BH, Ku PW, Stamatakis E. Can physical activity eliminate the mortality risk associated with poor sleep? A 15-year follow-up of 341,248 MJ Cohort participants. J Sport Health Sci. 2021. doi:10.1016/j.jshs.2021.03.001

55. Zuo H, Shi Z, Yuan B, et al. Interaction between physical activity and sleep duration in relation to insulin resistance among non-diabetic Chinese adults. BMC Public Health. 2012;12:247. doi:10.1186/14712458-12-247

56. He L, Biddle SJH, Lee JT, et al. The prevalence of multimorbidity and its association with physical activity and sleep duration in middle aged and elderly adults: a longitudinal analysis from China. Int J Behav Nutr Phys Act. 2021;18(1):77. doi:10.1186/s12966-02101150-7

57. Grandner MA, Drummond SP. Who are the long sleepers? Towards an understanding of the mortality relationship. Sleep Med Rev. 2007;11(5):341-360. doi:10.1016/j.smrv.2007.03.010

58. Smagula SF, Koh WP, Wang R, Yuan JM. Chronic disease and lifestyle factors associated with change in sleep duration among older adults in the Singapore Chinese Health Study. J Sleep Res. 2016;25 (1):57-61. doi:10.1111/jsr.12342
59. He L, Yang N, Ping F, et al. Long sleep duration is associated with increased high-sensitivity C-reactive protein: a Nationwide Study on Chinese Population. Diabetes Metab Syndr Obes. 2020;13:4423-4434. doi:10.2147/DMSO.S265465

60. Albert MA, Glynn RJ, Ridker PM. Effect of physical activity on serum C-reactive protein. Am J Cardiol. 2004;93(2):221-225. doi:10.1016/j.amjcard.2003.09.046

61. Loprinzi PD, Abbott K. Association of diabetic peripheral arterial disease and objectively-measured physical activity: NHANES 20032004. J Diabetes Metab Disord. 2014;13:63. doi:10.1186/2251-658113-63

62. Ashor AW, Lara J, Siervo M, et al. Exercise modalities and endothelial function: a systematic review and dose-response meta-analysis of randomized controlled trials. Sports Med. 2015;45(2):279-296. doi:10.1007/s40279-014-0272-9

63. Laufs U, Wassmann S, Czech T, et al. Physical inactivity increases oxidative stress, endothelial dysfunction, and atherosclerosis. Arterioscler Thromb Vasc Biol. 2005;25(4):809-814. doi:10.1161/ 01.ATV.0000158311.24443.af

64. Sabbahi A, Arena R, Elokda A, Phillips SA. Exercise and hypertension: uncovering the mechanisms of vascular control. Prog Cardiovasc Dis. 2016;59(3):226-234. doi:10.1016/j.pcad.20 16.09.006

65. Lee O, Lee DC, Lee S, Kim YS, Tauler P. Associations between physical activity and obesity defined by waist-to-height ratio and body mass index in the Korean population. PLoS One. 2016;11(7): e0158245. doi:10.1371/journal.pone.0158245

66. Colberg SR, Sigal RJ, Yardley JE, et al. Physical activity/exercise and diabetes: a position statement of the American Diabetes Association. Diabetes Care. 2016;39(11):2065-2079. doi:10.2337/dc16-1728
Risk Management and Healthcare Policy

\section{Publish your work in this journal}

Risk Management and Healthcare Policy is an international, peerreviewed, open access journal focusing on all aspects of public health, policy, and preventative measures to promote good health and improve morbidity and mortality in the population. The journal welcomes submitted papers covering original research, basic science, clinical \& epidemiological studies, reviews and evaluations, guidelines, expert opinion and commentary, case reports and extended reports. The manuscript management system is completely online and includes a very quick and fair peer-review system, which is all easy to use. Visit http://www.dovepress.com/testimonials.php to read real quotes from published authors. 